Revue des patrimoines

\title{
La Ferme et le Village radieux de Le Corbusier. Nouvelle déclinaison du principe d'équilibre entre l'individuel et le collectif
}

\section{Gilles Ragot}

\section{OpenEdition}

Journals

Édition électronique

URL : http://journals.openedition.org/insitu/10445

DOI : 10.4000/insitu. 10445

ISSN : 1630-7305

Éditeur

Ministère de la culture

Référence électronique

Gilles Ragot, «La Ferme et le Village radieux de Le Corbusier. Nouvelle déclinaison du principe d'équilibre entre l'individuel et le collectif », In Situ [En ligne], 21 | 2013, mis en ligne le 17 juillet 2013, consulté le 30 avril 2019. URL : http://journals.openedition.org/insitu/10445 ; DOI : 10.4000/ insitu. 10445

Ce document a été généré automatiquement le 30 avril 2019.

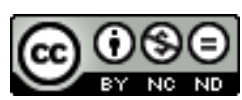

In Situ Revues des patrimoines est mis à disposition selon les termes de la licence Creative Commons Attribution - Pas d'Utilisation Commerciale - Pas de Modification 4.0 International. 


\title{
La Ferme et le Village radieux de Le Corbusier. Nouvelle déclinaison du principe d'équilibre entre l'individuel et le collectif
}

\author{
Gilles Ragot
}

\section{La Ferme et le Village radieux parties prenantes de la Ville radieuse}

1 À l'automne 1935, Le Corbusier publie La Ville radieuse, son quinzième livre, le troisième consacré aux questions d'urbanisme ${ }^{1}$. La Ville radieuse représente l'image paradigmatique de l'urbanisme progressiste auquel les allemands Ludwig Hilbersheimer et Walter Gropius apportent également une contribution majeure. Cet urbanisme progressiste s'oppose radicalement à la tendance culturaliste dont l'ouvrage de référence est Der Stätdebau (L'art de bâtir les villes), publié dès 1889 par l'autrichien Camillo Sitte, ou au courant anti-urbain et "naturaliste» qu'incarne Broadacre City de Frank Lloyd Wright, où l'architecte américain exposait les conceptions urbaines qu'il avait développées dans son ouvrage The Disappearing City, paru trois ans plus tôt, en 1932. La Ville radieuse consacre la naissance de l'urbanisme fonctionnaliste, dont les CIAM, et Le Corbusier lui-même, tireront le vademecum connu sous le nom de Chartes d'Athènes, publié en 1943.

Si cet ouvrage est régulièrement cité par les urbanistes, les historiens et les critiques de l'urbanisme, on oublie souvent que la septième partie de ce manifeste de l'urbanisme réformateur - la dernière avant la conclusion-est paradoxalement consacrée à la Réorganisation rurale qui comprend la présentation de ses projets de ferme et de village radieux. La Ville radieuse est une compilation de textes rédigés entre 1928 et 1935, dont la majeure partie a déjà été publiée à l'occasion de congrès ou dans diverses revues en particulier Plans, L'Homme réel et Prélude ${ }^{2}$. C'est notamment le cas de l'essentiel des textes qui composent la partie consacrée à la réorganisation rurale, déjà publiés dans L'Homme 
réel, Prélude et Architecture d'aujourd'hui ${ }^{3}$. Les projets de Ferme radieuse et de Village radieux ont également déjà été présentés dans les volumes deux et trois de L'Euvre complète, parus respectivement en 1934 et $1938^{4}$. Mais la place que Le Corbusier accorde à cette question de réorganisation rurale dans la septième partie de La Ville radieuse en fait à la fois la première présentation aussi développée de ces projets d'aménagement ruraux, témoignant de l'importance que Le Corbusier leur accorde au sein d'une vision élargie de la notion d'urbanisme qu'il qualifie « d'urbanisme total », en conclusion de son ouvrage5.

3 L'intérêt que Le Corbusier porte à la Réorganisation agraire est à la fois circonstanciel et fondamental. Le Corbusier et son cousin Pierre Jeanneret, avec lequel il est associé pendant l'entre-deux-guerres, conçoivent le projet de Ferme radieuse, puis celui de Village radieux à la suite des sollicitations de Norbert Bézard, ouvrier agricole ${ }^{6}$ à Piacé dans la Sarthe ${ }^{7}$. Norbert Bézard, ancien combattant, militant de la ruralité, a rencontré Le Corbusier par l'intermédiaire de Philippe Lamour et du docteur Pierre Winter liés comme l'architecte aux milieux planistes de l'entre-deux-guerres ${ }^{8}$. Jean-Claude Vigato et Daniel Le Couédic ont parfaitement montré que le "groupuscule politique » qui édita Plans et Prélude partageait avec Le Corbusier des convictions antiparlementaristes et corporatistes ${ }^{9}$. Comme nombre de français appartenant à la droite et à l'extrême droite, mais également à la gauche coopérative inspirée de Fourrier, Proudhon et Sorel, Le Corbusier dénonce le capitalisme et une société de gaspillage et de consommation de biens qu'il juge inutiles et annonce le « crépuscule de l'argent $»^{10}$. L'antiparlementarisme est un leitmotiv des revues Plans (1931-1932) et Prélude (1933-1936). Selon Le Couédic la tonalité de la revue Plans, nourrie des thèses de Pierre-Joseph Proudhon et de Georges Sorel était celle "d'un socialisme autoritaire appelant un ordre et une organisation assurés par une base constituée en coopérative» ${ }^{11}$. Pour Zeev Sternhell, auteur d'un ouvrage intitulé Ni droite ni gauche. L'idéologie fasciste en France, Plans est une «publication avant-gardiste, moderniste, type quasi parfait d'un fascisme orienté vers la technique, vers le gratte-ciel, vers la ville de Walter Gropius et de Le Corbusier, vers l'art de Fernand Léger, mais aussi vers une société organique harmonieuse, la société de "l'homme réel" " ${ }^{12}$. Socialisme autoritaire, ou fascisme techniciste, la définition des mouvances politiques de l'entre-deux-guerres ne saurait être donnée à l'aune du sens que l'on attribue à ces termes aujourd'hui. Jean-Louis Cohen parle du pullulement « des revues d'idées et de culture liées aux différentes familles politiques, phénomène caractéristique du "nonconformisme" de l'entre-deux-guerres ${ }^{13} »$. L'idéologie corporatiste dont se réclament les rédacteurs de Plans et de Préludes, dont Le Corbusier, connait une grande vogue dans les années 1930. Le fondement de cette idéologie est profondément antilibéral. Elle réunit des sympathisants d'extrême droite et des socialistes non marxistes qui, par la voie de la planification, en appellent à une mise en ordre de la société menée par un pouvoir fort où des technocrates ingénieurs éclairés joueraient un rôle majeur. Comme Le Corbusier, ils se méfient de la stérilité et de l'improductivité des institutions démocratiques et en appellent à «l'Autorité » servie par des hommes reconnus non pour leur appartenance politique, mais pour leur excellence dans un domaine de compétence reconnu. Avec $\mathrm{La}$ Ville radieuse, Le Corbusier s'affiche parmi ces spécialistes, dans son domaine de prédilection: l'architecture et l'urbanisme. La collaboration avec Norbert Bézard lui permet de s'immiscer dans celui de «l'urbanisme rural ». 


\section{Un principe d'harmonie entre l'individuel et le collectif}

4 Les projets de Ferme et de Village radieux, comme celui de la Ville radieuse, de même que le concept «d'unité » qui naît à cette époque sous diverses formes - unité d'habitation, unité de loisirs, unité industrielle, unité d'exploitation agricole ou unité rurale - relèvent d'une vision plus globale d'aménagement du territoire et de planification que portent précisément ces milieux planistes. Voir les sites: Fondation Le Corbusier (rubrique Archives, sous-rubrique Projets) et http://www.piaceleradieux.com/bezard-lecorbusierfermeradieuse.php.

5 Cette volonté impérieuse, totalitaire au sens didactique du terme puisqu'il rend compte, ou tente de rendre compte, des éléments d'un phénomène, qui englobe ou tente d'englober la totalité des éléments d'un ensemble, est profondément ancrée dans la pensée corbuséenne. Elle prend la forme d'un fonctionnalisme biologique qui s'attache à relier l'élément au tout, à assurer l'harmonie entre tous les organes qui constituent un organisme vivant : en l'occurrence la société des hommes.

6 Le docteur Winter, déjà cité pour sa participation aux milieux planistes, qualifiait Le Corbusier de biologiste. "C'est, écrit le docteur Winter, en partant de la biologie élémentaire et de l'observation de la vie moderne, avec la préoccupation constante de satisfaire les besoins et d'équilibrer l'emploi du temps de l'homme d'aujourd'hui, que Le Corbusier et Pierre Jeanneret ont peu à peu développé leur magnifique programme ${ }^{14}$ ».

7 Ce n'est pas tant la prise en compte, comme le dit le docteur Winter, des bonnes « doses de lumière,... de l'eau, du silence,... de la culture physique $»^{15}$, qui fonde l'approche biologique de Le Corbusier, qu'une démarche méthodologique reposant sur une capacité d'analyse et une volonté de diagnostic préalables à toute intervention projectuelle.

Ce souci trouve précisément un écho dans les écrits de Le Corbusier lorsqu'il évoque sa descente en analyse et son travail de laboratoireen1922, au cours de la conférence intitulée « Un homme = une cellule, des cellules = la ville ${ }^{16}$. De cette double équation émerge un principe corbuséen fondamental : celui de l'harmonie entre l'individuel et le collectif, entre la cellule et l'organisme. Les sources de ce principe sont connues et reconnues par l'architecte lui-même : ce sont celles du couvent et plus particulièrement de la Chartreuse d'Ema, que Le Corbusier visite dès 1906, celle du fouriérisme que Le Corbusier cite à plusieurs reprises dans ses textes ou notes personnelles, et celle du "paquebot", modèle avoué dès l'article "Des yeux qui ne voient pas: les paquebots " publié en mai 1921 dans L'Esprit nouveau ${ }^{17}$.

9 À la Chartreuse d'Ema de Galluzo, comme dans le modèle du phalanstère fouriériste ou du paquebot transatlantique, c'est le principe organisationnel qui séduit le jeune Jeanneret. La cellule conserve son indépendance au sein du collectif. Mais, son existence, comme l'identité du moine, du phalanstérien, ou du passager des paquebots, n'a de sens qu'au sein de la collectivité ${ }^{18}$. Cette observation, faite dès 1906, trouve une traduction théorique dans la phrase écrite dix-huit ans plus tard pour le dernier chapitre de l'ouvrage Urbanisme. «Un ensemble, écrit Le Corbusier en 1925, est fait d'infiniment petites parties qui sont parfaites, qui sont elles-mêmes en un ensemble, un système réduit à l'essentiel. La cellule conditionne l'ensemble; la cellule doit être un système pur. L'ensemble ne vit que par la cellule. La cellule prend son efficacité du fait qu'elle est admissible dans l'ensemble ${ }^{19}$.» 
10 Ce chapitre qui clôt Urbanisme est entièrement illustré de schémas ou de coupes de biologie végétale ou animale empruntés au biologiste Gaston Bonnier dans son ouvrage intitulé L'enchaînement des organismes ${ }^{20}$.

11 Cette citation pose fondamentalement, le principe politique, technique et vital de la Ferme radieuse, du Village coopératif et des multiples déclinaisons de ces projets. L'analogie biologique apporte une dimension fondamentale, qui permet d'inscrire la Ferme radieuse dans une œuvre globale et cohérente.

12 De cette quête d'harmonie entre la cellule et la ville, entre l'individu et la société, Le Corbusier dégage un principe d'équilibre entre l'individuel et le collectif où prime la liberté individuelle. Tout au long de La Ville radieuse, à vingt reprises, Le Corbusier ne cesse de répéter que le respect de «la liberté individuelle» est à la base d'un nouveau contrat social. «Dans la ville, la liberté individuelle constitue la pierre angulaire de la doctrine $^{21}$. » Mais une liberté individuelle qui se construit dans la recherche constante d'un équilibre parfait avec le collectif. C'est dans la quête de cette harmonie fondamentale que se fonde le projet urbanistique et rural corbuséen, qui se différencie autant du modèle libéral que du modèle bolchevique.

13 Moins de dix ans plus tard, Le Corbusier fera de ce principe l'article 2 de la Charte d'Athènes: "Juxtaposée à l'économique, au social et au politique, les valeurs d'ordre psychologiques et physiologiques attachées à la personne humaine, introduisent dans le débat des préoccupations d'ordre individuel et d'ordre collectif. La vie ne s'épanouit que dans la mesure où s'accordent les deux principes contradictoires qui régissent la personnalité humaine : l'individuel et le collectif ${ }^{22}$."

\section{Plaidoyer pour un nouveau statut foncier}

14 Ce principe clé de la pensée corbuséenne régit tout autant le fonctionnement des futures unités d'habitation, que celui de la Ville radieuse, ou des Fermes et Villages radieux. La réalisation de ces projets à une échelle urbaine ou rurale suppose un nouveau statut du foncier. Le Corbusier propose un remembrement intégral, non seulement urbain mais rural. Il préconise une « loi préalable et indispensable de remembrement du sol des villes et des campagnes ${ }^{23}$ ", mais n'appelle jamais à une confiscation ou à une nationalisation de la propriété individuelle. Il consacre un chapitre entier à la "Mobilisation du sol ", où il précise que « disposer ne signifie pas anéantir la propriété, ni voler, ni spolier. Cela signifie mettre en valeur les richesses et les ressources de la terre pour le bienfait des hommes $^{24} »$. Le dispositif proposé ressemble à celui du remembrement urbain qui sera généralisé lors de la reconstruction des villes dévastées par la Seconde Guerre mondiale, mais Le Corbusier s'en remet aux spécialistes pour trouver la solution juridique ad hoc: «Que les juristes cherchent la formule! Mobiliser n'est pas nationaliser. C'est rendre solidaire ${ }^{25}$. " Cet outil que Le Corbusier envisageait déjà pour la réalisation du Plan Voisin, s'impose en 1935 comme l'une des conditions de La Ville radieuse, à tel point, que, lors de la réédition de l'ouvrage en 1964, il ajouta en page 2 un encadré où il en appelait à la «Mobilisation du sol». Entre temps, ce dispositif juridique, économique et politique figurait en bonne place dans la Charte d'Athènes que Le Corbusier avait publié en 1943.

15 L'objectif de Norbert Bézard, initiateur des projets corbuséens de Ferme et de Ville radieuse n'est pas différent: il s'agit de mettre en commun les moyens au sein d'un village coopératif: regrouper les terres, acquérir et utiliser en commun le matériel 
coûteux que les techniques modernes et machinistes de l'agriculture imposent. «Il faut donner la terre aux paysans! Mais la leur donner de façon conditionnelle. Posséder la terre est en fait un privilège : face à ce privilège, il faut mettre des obligations qui empêchent de créer une nouvelle caste féodale de possédants. Il n'est donc que d'encadrer ces possédants dans des organisations syndicales régies par des modalités d'exploitation qui limitent le droit de propriété à la fonction parfaitement remplie dans le sein de cette collectivité, tout en respectant les vraies libertés essentielles à la vie normale de l'individu et de la famille.

«C'est justement ce réajustement total qui nous permettra de remembrer exactement les parcelles et de grouper les cultures de façon à pouvoir employer les méthodes de la grande culture avec un outillage moderne supprimant le salariat aux champs, et abaissant le prix de revient. Le syndicat communal a le moyen de posséder les coûteuses machines motorisées (limitation exacte du matériel aux besoins), alors que le petit fermier ne peut pas les posséder, faute de capitaux. C'est dans une certaine mesure la collectivisation de la production, mais à l'inverse des méthodes communistes, cette collectivisation se borne à la propriété collective des grosses machines, magasins, silos, atelier municipal, etc. Chaque famille a donc bien à elle le produit de sa terre, mais avec les avantages obtenus par l'exploitation par moyens communs, voilà tout ${ }^{26}$."

17 Sous le terme de " coopération intégrale » Bézard propose une exploitation collective et rationnelle des terres dont chacun conserve la propriété et l'usufruit. Le principe trouve écho dans Histoire de la campagne française, que l'historien ruraliste Gaston Roupnel a publié en $1932^{27}$ : «Sans avoir recours au remembrement, sans modifier la répartition de la propriété, il existe un moyen d'adapter la répartition de la propriété, il existe un moyen d'adapter notre campagne actuelle aux nouveaux procédés d'exploitation. L'association est susceptible de produire les mêmes effets que le remembrement. Dans un bloc composé de plusieurs parcelles, la propriété peut rester morcelée : il suffit que l'exploitation en soit unique, c'est-à-dire assurée par des machines et par une maind'œuvre au service de la collectivité. Le village tendrait à devenir une association d'exploitants et une coopérative de production. On en reviendrait aux temps de l'exploitation collective ; on en reviendrait aux temps originels où chacun était au service de tous.» Cet extrait sera repris par Le Corbusier à l'appui du chapitre "L'unité d'exploitation agricole " publié en 1945 dans Les Trois Établissements humains ${ }^{28}$.

D'autres thèmes communs rapprochent les écrits de Roupnel, de Le Corbusier et de Bézard: le machinisme et la mécanisation, l'aspiration au confort moderne dans les campagnes, mais aussi la quête de l'équilibre entre l'individuel et le collectif. Gaston Roupnel écrit à ce sujet en 1932 : «Les associations et les coopératives, qui permettront de cultiver en grand et de moderniser les industries de la ferme, introduiront dans nos campagnes le double bénéfice de la spécialisation et de la concentration de la terre, tout en respectant le fractionnement de la propriété, l'indépendance de la production et les initiatives de la vie individuelle ${ }^{29}$. »

19 Enfin, tous partagent le même intérêt pour les voies de communication considérées comme l'outil premier de l'aménagement du territoire qui unit la ville et le monde rural dans une même vision préfigurant un monde globalisé pour un homme universel ${ }^{30}$. Plus que de simples réponses à une sollicitation de Norbert Bézard, les projets élaborés pour l'essentiel entre 1933 et 1937 par Le Corbusier et Pierre Jeanneret s'inscrivent donc pleinement dans un projet global d'urbanisme total. Les architectes élaborent différentes propositions guidées par des principes semblables: une étude de Ferme radieuse; une 
étude de Village coopératif ou Village radieux; une adaptation de ce Village type au site de Piacé où Norbert Bézard habite et travaille ; une Unité d'exploitation agricole en 1944.

\section{Un urbanisme rural fonctionnaliste} l'habitation du paysan: «Dans la "Ferme radieuse", le logis est indépendant des écuries, des étables, des hangars et de la grange : il est situé sur l'axe de services généraux, comme un poste de commandement ${ }^{33}$.» Sur le plan architectural, Le Corbusier n'invente pas un type nouveau d'habitat rural. Il adapte au monde rural un type d'habitat urbain, le modèle de maisons qu'il vient d'élaborer dans le cadre de la loi Loucheur en 1929 et qui reprend lui-même les principes de la Maison minimum type CIAM de $1929^{34}$. Le Corbusier affirme ainsi le caractère global de sa vision de l'aménagement du territoire et se montre par ailleurs fidèle à sa pratique de recyclage de ses concepts ou de ses projets.

Ce transfert d'un modèle type de la ville à la campagne, du monde ouvrier au monde paysan, montre encore que le logis paysan ne s'inscrit dans aucune vision romantique, régionaliste ou nostalgique, mais qu'il s'agit bien pour Le Corbusier, comme pour Norbert Bézard, d'offrir au paysan, comme à l'ouvrier ou au petit employé de bureau, la même architecture. L'universalité recherchée par Le Corbusier se situe ainsi à plusieurs niveaux :

- universalité culturelle dans la recherche d'une architecture supra-culturelle dont le purisme des années 1920 était la plus authentique expression ; 
- universalité géographique dans la recherche d'une architecture hors sol dont le pilotis est l'image paradigmatique ;

- universalité sociale dans la recherche d'une architecture identique pour tous, sans classe. principe standard d'ossature métallique couverte de voûtes surbaissées «de mince ciment garnies de terre végétales ». Le module métallique confère harmonie, rythme et assure une production au moindre coût. Le choix du métal, plutôt que celui du béton, confirme la préférence accordée pendant tout l'entre-deux-guerres par Le Corbusier et Pierre Jeanneret au métal dans tous leurs projets majeurs. On retrouve également dans les formes modulaires adoptées une nouvelle déclinaison des maisons MONOL conçues dès $1919^{37}$.

\section{Des propositions sans lendemain} Le Corbusier à publier régulièrement cette étude, prouve sa foi et son engagement pour ce projet. Il reprend mais ne développe plus ces concepts de ferme et de villages radieux après la Seconde Guerre mondiale. Sur le plan architectural, il décline le modèle de la maison du paysan hérité de la loi Loucheur en différentes versions sur des sites variés pour des commandes incertaines dont aucune n'aboutira. Parmi celles-ci, la plus notable est celle de la maison rurale ou logis paysan, à Chessy (1955-1957), conçue avec le concours de Jean Prouvés. Mais c'est surtout en tant qu'outil d'aménagement du territoire que les réflexions engagées dans les années 1930 perdureront.

31 L'unité paysanne grandeur conforme représente pour l'architecte un outil d'aménagement du territoire au même titre que l'unité d'habitation grandeur conforme sur le plan de l'urbanisme. Comme le montre le dessin FLC 20336, non daté mais probablement des années 1930. Le Village et la Ferme radieuse sont des outils au service d'une mise en ordre du paysage: le territoire est carroyé selon l'unité de base d'une 
ferme radieuse. Le Corbusier reprend un dispositif semblable, mais plus proche du principe du rhizome pour élaborer en 1944 le projet d'Unité d'exploitation agricole ${ }^{39}$.

C'est cette dimension qui rejaillit encore dans l'ouvrage déjà cité, publié en 1945 : Les Trois Établissements humains. L'Unité d'exploitation agricole est précisément l'un de ces trois établissements qui fondent le territoire aux côtés de la Cité linéaire industrielle et la Cité radioconcentrique des échanges.

Mais Le Corbusier n'aura pas l'occasion d'expérimenter ce nouveau dispositif d'aménagement du sol, malgré plusieurs tentatives de s'immiscer dans de grands projets lancés par l'État après la Seconde Guerre mondiale. Il sollicite notamment en 1957 Philippe Lamour, ancien fondateur de Prélude et futur initiateur de la Datar. Il le retrouve président de la Reconversion du Bas Rhône et du Languedoc ${ }^{40}$ et lui propose d'intégrer des fermes et des villages radieux dans ce grand projet. En vain. L'État devenu planificateur ne fit jamais appel à Le Corbusier et aucun projet de Ferme ou de Village radieux ne vit le jour.

\section{NOTES}

1. - LE CORBUSIER, La Ville radieuse. Paris : Éditions de l'Architecture d'aujourd'hui, 1935. Les deux premiers sont Urbanisme et Précisions sur un état présent de l'architecture et de l'urbanisme, publiés respectivement en 1925 et en 1930.

2. - Sur la genèse de La Ville radieuse on se reportera à l'article de RAGOT, Gilles. « Le Corbusier, La Ville radieuse ou le Despote", préface à la réédition du texte original de La Ville radieuse à paraître aux Éditions Parenthèses en 2013.

3. - Le chapitre 2, «La ferme radieuse " a déjà été publié dans Prélude $\mathrm{n}^{\circ} 14$ en décembre 1934, sous le titre " Réorganisation agraire. 1933-1934. La "Ferme radieuse", le "Village radieux" », et dans L'Homme réel, $\mathrm{n}^{\circ}$ 4, avril 1934, sous le titre "La Ferme radieuse. Le Village radieux », ainsi que dans Architecture d'aujourd'hui, en février 1935.

4. - LE CORBUSIER et JEANNERET, Pierre. Euvre compète de 1929-1934. Zurich : Éditions Girsberger, 1984 (1 $1^{\text {re }}$ édition par Willy Boesiger, Les Éditions d'Architecture, 1934), p. 186-191. CEuvre complète 1934-1938. Zurich : Éditions Girsberger, 1975 ( $1^{\text {re }}$ édition par Max Bill, Les Éditions d'Architecture), p. 104-115.

5. - «Urbanisme total » est le premier chapitre de la $8^{\mathrm{e}}$ partie, conclusive, de La Ville radieuse.

6. - Norbert Bézard (1896-1956) exerce diverses professions - apiculteur, ouvrier agricole, ouvrier maçon à Piacé dans la Sarthe, puis ouvrier chez Renault à Paris, avant de consacrer la fin de sa vie à la céramique. Voir le site dédié au projet de Le Corbusier pour Piacé ; < lien vers : http:// www.piaceleradieux.com/ >.

7. - La correspondance entre Bézard et ses architectes, conservée à la Fondation Le Corbusier, commence en 1933 et s'achève en 1939. Voir FLC H3-16 et L3-20.

8. - Philippe Lamour (1903-1992), avocat et homme politique, appartient aux milieux fascistes avant la Seconde Guerre mondiale. Il est l'un des fondateurs de la revue Plans. Après la Seconde Guerre mondiale il fait une carrière de grand technocrate au service de la $\mathrm{IV}^{\mathrm{e}}$ et de la $\mathrm{V}^{\mathrm{e}}$ République au service de la modernisation de l'agriculture et de l'aménagement du territoire. Pierre Winter, né en 1891, médecin, chirurgien en chef de la faculté de médecine de Paris, est un 
proche de Le Corbusier dès ses premiers articles dans la revue L'Esprit nouveau. Il se réclame politiquement de l'autorité de Philippe Lamour.

9. - VIGATO, Jean-Claude. L'architecture régionaliste. France. 1890-1950. Paris : Éditions Norma, coll. «Essais », 1994. LE COUÉDIC, Daniel. «Les fondements idéologiques du planisme de Le Corbusier ». Urbanisme, $\mathrm{n}^{\circ} 223$, février 1988, p. 56-63. Sur les positionnements politiques de Le Corbusier, voir également, COHEN, Jean-Louis. "Droite-gauche: "invite à l'action" ". Encyclopédie Le Corbusier. Paris : Éditions du Centre Georges Pompidou, 1987, p. 309-313.

10. - LE CORBUSIER. La Ville radieuse. Paris : Éditions de l'Architecture d'aujourd'hui, 1935, p. 7.

11. - LE COUÉDIC, Daniel. «Les fondements idéologiques du planisme de Le Corbusier». Urbanisme, $\mathrm{n}^{\circ} 223$, février 1988, p. 56-63.

12. - STERNHELL, Zeev. Ni droite ni gauche. L'idéologie fasciste en France. Paris : Le Seuil, 1983.

13. - COHEN, Jean-Louis. "Droite-gauche: “invite à l'action” ». Encyclopédie Le Corbusier. Paris : Éditions du Centre Georges Pompidou, 1987, p. 309-313.

14. - WINTER, Pierre. «Le Corbusier ». L'Architecture d'aujourd'hui, spécial Le Corbusier, $\mathrm{n}^{\circ} 10$, 1933, p. 9.

15. - Ibid.

16. - LE CORBUSIER. Précisions sur un état présent de l'architecture et de l'urbanisme. Paris : Éditions Altamira, 1994 ( $1^{\text {re }}$ édition G. Crès et Cie, Paris, 1930), p 141.

17. - L'article est repris ensuite dans Vers une architecture, publié en 1923.

18. - Le Corbusier visite la Chartreuse d'Ema à Galluzo près de Florence lors de son voyage en Italie en 1906. Il est fasciné par l'organisation du couvent qui permet à chaque moine chartreux de s'isoler dans une maison individuelle dotée d'un jardin privatif ouverte sur le cloître et de bénéficier des avantages et des services de la vie collective que leur offre la communauté monastique.

19. - LE CORBUSIER. Urbanisme. Paris : Collection de l'Esprit nouveau, 1925, p. 287.

20. - BONNIER, Gaston. Histoire naturelle de la France. Première partie. Généralités : L'enchaînement des organismes. Paris : Éditions les Fils d'Émile Deyrolles, s.d. G. Bonnier était membre de l'Institut et professeur à la Sorbonne. Un exemplaire de cet ouvrage figure dans la bibliothèque personnelle de Le Corbusier.

21. - LE CORBUSIER. La Ville radieuse. Paris : Éditions de l'Architecture d'aujourd'hui, 1935, p. 96.

22. - LE CORBUSIER. La Charte d'Athènes. Avec un discours liminaire de Jean Giraudoux. Paris : Éditions de Minuit, 1957 ( $1^{\text {re }}$ édition en 1943 aux éditions Plon), p. 20.

23. - LE CORBUSIER. La Ville radieuse. Paris : Éditions de l'Architecture d'aujourd'hui, 1935, p. 24.

24. - LE CORBUSIER. La Ville radieuse. Paris : Éditions de l'Architecture d'aujourd'hui, 1935, p. 189.

25. - LE CORBUSIER. La Ville radieuse. Paris : Éditions de l'Architecture d'aujourd'hui, 1935, p. 188.

26. - BEZARD, Norbert. « La terre aux paysans ». L’homme réel, $n^{\circ}$ 4, avril 1934, p. 38.

27. - L'ouvrage de Gaston Roupnel est dans la bibliothèque personnelle de Le Corbusier. G. Roupnel (1871-1946), professeur d'histoire à l'université de Dijon publie, Histoire de la campagne française. Paris : Éditions Grasset, 1932. Il est aussi romancier, auteur de Nono qui connût un vif succès en 1910. Le géographe Jean-Robert Pitte, auteur de l'Histoire du paysage français (Paris : Tallandier 1983), considère le travail de Roupnel de "plus intuitif que scientifique » (cf. op. cit, t. 2, p. 166).

28. - LE CORBUSIER. Les Trois Établissements humains. Paris: Éditions de L'Architecture d'aujourd'hui, 1945, p. 77-78. Cet ouvrage, est le fruit des travaux de l'Ascoral conduit notamment avec Norbert Bézard.

29. - ROUPNEL Gaston, op. cit, p. 208.

30. - ROUPNEL Gaston écrit dans Histoire de la campagne française, p. 293: « Chacun de ces petits villages qui commença par être tout seul sur terre, s'éveilla peu à peu au Monde avec sa campagne solitaire. Il s'anima d'abord à la vie de tout le pays; il connut après la province ; ensuite la France. Et maintenant, c'est l'universelle volonté du Monde qui règne aujourd'hui sur 
ce site étroit et y dicte les mêmes volontés impérieuses qu'à toute la Terre. Des crises mondiales y décident de la prospérité du vignoble, de l'aisance et de l'activité de chaque logis. Tout sera dit de cette campagne, quand on aura montré que l'homme universel y est aussi entièrement contenu dans son histoire que dans ses destinées. »

31. - LE CORBUSIER. La Ville radieuse. Paris : Éditions de l'Architecture d'aujourd'hui, 1935, p. 327.

32. - Sur les projets d'abattoirs de Challuy et Garchizy et Bordeaux voir RAGOT, Gilles et DION, Mathilde. Le Corbusier en France. Paris : Éditions du Moniteur, coll. "Architextes », 1997 (1 $1^{\text {re }}$ éditions en 1987), p. 29-31.

33. - LE CORBUSIER. Sur les quatre routes. Paris : Denoël/Gonthier, 1970 ( $1^{\text {re }}$ édition NRF Gallimard, Paris, 1941), p. 105-106.

34. - Ce modèle présenté au CIAM de Francfort en 1929 a été réalisé en un unique exemplaire pour le pavillon du gardien de la villa Savoye (1928-1931) à Poissy.

35. - L'immeuble-villas est un modèle d'immeuble collectif alliant appartements individuels en duplex articulés sur un jardin intérieur, et services collectifs à disposition de tous les habitants, imaginé par Le Corbusier à partir de 1922. Voir RAGOT, Gilles et DION, Mathilde. Le Corbusier en

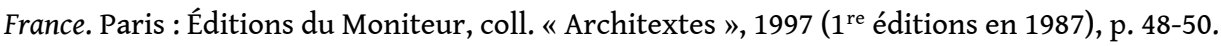

36. - BEZARD, Norbert, dans LE CORBUSIER. Les Trois Établissements humains. Paris : Éditions de L'Architecture d'aujourd'hui, 1945, p. 92.

37. - Sur les maisons Monol, voir RAGOT, Gilles et DION, Mathilde. Le Corbusier en France. Paris : Éditions du Moniteur, coll. « Architextes », 1997 (1 $1^{\text {re }}$ éditions en 1987), p. 32-33.

38. - Ces études sont les suivantes: Maisons pour ingénieurs et contremaîtres à Lannemezan, 1940 ; Maisons rurales ou logis paysan à Chessy, 1955-1956; Maisons montées à sec (MAS) ou "type Loucheur", à Lagny, 1955-1957. Aucun de ces projets ne fut réalisé. Voir RAGOT, Gilles et DION, Mathilde, Le Corbusier en France, op. cit., respectivement p. 265-266, 361-363 et 364.

39. - Voir en particulier le dessin FLC 28615, mai 1944. Projet d'Unité d'exploitation agricole.

40. - Voir en particulier la lettre de Le Corbusier à Norbert Bézard du 16 juin 1936, FLC H-3-16 pièce 14. Pour la tentative de participation de Le Corbusier à ce projet d'aménagement voir RAGOT, Gilles et DION, Mathilde, Le Corbusier en France, op. cit., p. 374-379.

\section{RÉSUMÉS}

Du début des années 1930 au milieu des années 1940, Le Corbusier décline plusieurs projets d'urbanisme rural : la ferme et le Village radieux, la maison rurale, l'unité d'exploitation agricole. Toutes ces études, dont aucune ne sera réalisée, s'inscrivent dans une réflexion plus vaste sur un « urbanisme total» et sur la question de l'aménagement du territoire qui suppose un nouveau statut du foncier. Les propositions corbuséennes, inspirées des travaux du géographe Gaston Roupnel, participent encore de la réflexion des milieux planistes dont il est l'un des plus fervents défenseurs à l'aube de la Seconde Guerre mondiale.

From the beginning of the thirties to the mid-forties, Le Corbusier presented a number of rural planning projects: the radiant farm and village, the rural house, the farming unit. All these studies, none of which were ever put into practice, are registered in a much wider treatise on "total urbanism" and on the question of territorial planning which implies a new status for property. Le Corbusier's proposals, inspired by the works of the geographer Gaston Roupnel, 
again share in the deliberations of the planning communities of which he was one of the most fervent defenders at the dawn of the Second World War.

INDEX

Mots-clés : Le Corbusier, Norbert Bézard, Gaston Roupnel, Philippe Lamour, Ferme radieuse, Village radieux, Ville Radieuse, aménagement du territoire, milieux planistes, planification

\section{AUTEUR}

\section{GILLES RAGOT}

Professeur à l'École nationale supérieure d'architecture et de paysage de Bordeaux. mathilde.dion@wanadoo.fr 